\title{
Defect Formation beyond Kibble-Zurek Mechanism and Holography
}

\author{
Paul M. Chesler \\ Department of Physics, Harvard University, Cambridge, Massachusetts 02139, USA \\ Antonio M. García-García ${ }^{\dagger}$ \\ TCM Group, Cavendish Laboratory, University of Cambridge, J. J. Thomson Avenue, \\ Cambridge CB3 OHE, United Kingdom \\ Hong Liu \\ Center for Theoretical Physics, MIT, Cambridge, Massachusetts 02139, USA
}

(Received 16 September 2014; revised manuscript received 5 February 2015; published 14 May 2015)

\begin{abstract}
We study the dynamic after a smooth quench across a continuous transition from the disordered phase to the ordered phase. Based on scaling ideas, linear response, and the spectrum of unstable modes, we develop a theoretical framework, valid for any second-order phase transition, for the early-time evolution of the condensate in the broken phase. Our analysis unveils a novel period of nonadiabatic evolution after the system passes through the phase transition, where a parametrically large amount of coarsening occurs before a well-defined condensate forms. Our formalism predicts a rate of defect formation parametrically smaller than the Kibble-Zurek prediction and yields a criterion for the breakdown of Kibble-Zurek scaling for sufficiently fast quenches. We numerically test our formalism for a thermal quench in a $(2+1)$ dimensional holographic superfluid. These findings, of direct relevance in a broad range of fields including cold atom, condensed matter, statistical mechanics, and cosmology, are an important step toward a more quantitative understanding of dynamical phase transitions.
\end{abstract}

DOI: 10.1103/PhysRevX.5.021015

\section{INTRODUCTION}

Driving a system smoothly from a disordered to an ordered phase unveils the rich, and still poorly understood, phenomenology of dynamical phase transitions, a research theme of interest in vastly different fields. The Kibble-Zurek (KZ) mechanism (KZM) describes the spontaneous generation of topological defects when a system is driven through a second-order phase transition into the ordered phase [1-3]. Numerical simulations [4-14] have confirmed the spontaneous generation of defects and the scaling exponent of the defect density with the quench rate predicted by the KZM. The KZM has also been generalized to quantum phase transitions [15-17] and has been employed to compute correlation functions [18] in the scaling region.

Different experiments, with ion crystals [19,20], ultracold atomic gases [21-23], spin liquids [24], superconducting films [25], polariton superfluids [26], Josephson junctions

\footnotetext{
*pchesler@physics.harvard.edu

amg73@cam.ac.uk

"hong_liu@mit.edu
}

Published by the American Physical Society under the terms of the Creative Commons Attribution 3.0 License. Further distribution of this work must maintain attribution to the author(s) and the published article's title, journal citation, and DOI.
Subject Areas: Condensed Matter Physics,

Gravitation, String Theory

[27], and helium [28-30], have observed, with different levels of certainty, defect generation, but a really quantitative comparison with the predictions of the KZM is still missing.

Let us briefly review the KZM [31-33]. Consider a system with a second-order phase transition at temperature $T_{c}$, below which a symmetry is spontaneously broken and an order parameter $\psi$ develops a condensate. In equilibrium at temperature $T>T_{c}$, the correlation length $\xi_{\mathrm{eq}}$ and relaxation time $\tau_{\text {eq }}$ are related to the reduced temperature $\epsilon \equiv 1-\left(T / T_{c}\right)$ by

$$
\begin{aligned}
& \xi_{\text {eq }}=\xi_{s}|\epsilon|^{-\nu}, \\
& \tau_{\text {eq }}=\tau_{s}|\epsilon|^{-\nu z},
\end{aligned}
$$

for some scales $\xi_{s}, \tau_{s}$ and critical exponents $\nu, z$. Consider a quench from $T_{i}>T_{c}$ to $T_{f}<T_{c}$ with quench protocol

$$
\begin{aligned}
\epsilon(t) & =t / \tau_{Q}, \\
t & \in\left(t_{i}, t_{f}\right),
\end{aligned}
$$

where $t_{i}=\left(1-T_{i} / T_{c}\right) \tau_{Q}<0$ and $t_{f}=\left(1-T_{f} / T_{c}\right) \tau_{Q}>0$. The system can respond adiabatically to the change in temperature until $\tau_{\mathrm{eq}}(t) \sim|t|$. This condition defines the freeze-out time and length scale 


$$
t_{\text {freeze }}=\tau_{s}\left(\frac{\tau_{Q}}{\tau_{s}}\right)^{\nu z /(1+z \nu)}, \quad \xi_{\text {freeze }}=\xi_{s}\left(\frac{\tau_{Q}}{\tau_{s}}\right)^{\nu /(1+\nu z)} .
$$

During the interval $t \in\left(-t_{\text {freeze }}, t_{\text {freeze }}\right)$, the evolution of the system is essentially frozen. ${ }^{1}$ The correlation length $\xi_{\text {freeze }}$ then imprints itself on the state at $t=+t_{\text {freeze }}$.

The density of topological defects generated across the phase transition can then be estimated as

$$
\rho_{\mathrm{KZ}} \sim 1 / \xi_{\text {freeze }}^{d-D} \sim \tau_{Q}^{(d-D) \nu /(1+\nu z)},
$$

where $d$ is the number of the spatial dimensions and $D$ is the number of dimensions of a defect. While the KZM is only supposed to determine the density of defects up to an $O(1)$ factor, it often significantly overestimates the real density of defects observed in numerical calculations: One needs a "fudge" factor $f$ multiplying $\xi_{\text {freeze }}$ with $f=O(10)$ [33]. See also Ref. [34] for a recent discussion.

One motivation of this paper is to develop a formalism for describing the growth and coarsening of the order parameter after $t_{\text {freeze }}$ in a general critical system. Our analysis stresses a period of nonadiabatic evolution, before a well-defined condensate forms, where the system coarsens and the correlation length grows parametrically larger than $\xi_{\text {freeze }}$. In particular, we show that in many systems, including conventional superconductors and superfluid ${ }^{4} \mathrm{He}$, there could be a large logarithmic hierarchy between $t_{\text {freeze }}$ and the time scale we refer to as $t_{\text {eq }}$ when one can sensibly measure the density of defects. Thus, our analysis reconciles the need for a fudge factor $f$. Moreover, our analysis yields a new criterion for the breaking of the $\mathrm{KZ}$ scaling (1.4). Our discussion can also be applied without essential changes to quantum phase transitions. For definiteness, we will restrict discussion to thermal phase transitions throughout the paper.

A second motivation of this paper is to test the scaling predicted by the KZM and its refinement in strongly coupled systems using holographic duality. Holography equates certain systems of quantum matter without gravity to classical gravitational systems in one higher spatial dimension [35-37]. Hence, complicated many-body physics can be mapped onto a solvable numerical gravity problem. Some examples include Refs. [38-47] (see also Refs. [48,49] for a discussion of KZM for a holographic quantum quench). In this paper, we study the KZM in a holographic superfluid in $2+1$ spacetime dimensions. Our gravity calculation will provide a first check of $\mathrm{KZ}$ scaling in a strongly coupled system without quasiparticles and

\footnotetext{
${ }^{1}$ Strictly speaking, one should distinguish $t_{\text {freeze }}^{>}$for $T>T_{c}$ and $t_{\text {freeze }}^{<}$for $T<T_{c}$ as they can differ by an $O(1)$ constant. We will suppress such differences for notational simplicities.
}

will verify key features of the coarsening physics discussed in the next section. ${ }^{2}$

\section{FAR-FROM-EQUILIBRIUM COARSENING}

\section{A. Unstable critical modes}

We now develop a formalism to describe a period of nonadiabatic growth of the order parameter $\psi$ after $t_{\text {freeze }}$. The seeds for condensate growth come from thermal and quantum fluctuations, whose effects on the macroscopic evolution of $\psi$ can be described in terms of a stochastic source $\varphi$ for $\psi$. In the IR, the statistics of the fluctuations in $\varphi$ read

$$
\left\langle\varphi^{*}(t, \boldsymbol{x}) \varphi\left(t^{\prime}, \boldsymbol{x}^{\prime}\right)\right\rangle=\zeta \delta\left(t-t^{\prime}\right) \delta\left(\boldsymbol{x}-\boldsymbol{x}^{\prime}\right),
$$

where $\zeta$ is a (weakly) temperature-dependent constant.

Let $\psi(t, \boldsymbol{q})$ and $\varphi(t, \boldsymbol{q})$ be the Fourier-transformed order parameter and noise, respectively. At early times, $\psi(t, \boldsymbol{q})$ is small and can be described by linear response

$$
\psi(t, \boldsymbol{q})=\int d t^{\prime} G_{R}\left(t, t^{\prime}, q\right) \varphi\left(t^{\prime}, \boldsymbol{q}\right),
$$

where $G_{R}\left(t, t^{\prime}, q\right)$ is the retarded $\psi$ correlator. Statistical homogeneity and isotropy imply $G_{R}$ only depends on $q=|\boldsymbol{q}|$. The regime of validity for the linear response will be discussed below. To elucidate the growth of $\psi$ and to extract the time evolution of the correlation length after $t_{\text {freeze }}$, we study the evolution of the correlation function

$$
C(t, \boldsymbol{r}) \equiv\left\langle\psi^{*}(t, \boldsymbol{x}+\boldsymbol{r}) \psi(t, \boldsymbol{x})\right\rangle .
$$

Averaging over the noise (2.1), we find

$$
C(t, q)=\int d t^{\prime} \zeta\left|G_{R}\left(t, t^{\prime}, q\right)\right|^{2}
$$

As the dynamics is essentially frozen between $\left(-t_{\text {freeze }}, t_{\text {freeze }}\right)$, at $t \sim t_{\text {freeze }}$, the system is in a supercooled state for which the leading time dependence of $G_{R}$ can be obtained by analytically continuing to below $T_{c}$ the equilibrium-retarded correlator $G_{\text {eq }}$ above $T_{c}$. Close to $T_{c}$, the time dependence of $G_{\text {eq }}(t, q)$ should be dominated by the leading pole $\mathfrak{w}_{0}(q)$ (the critical mode) of $G_{\text {eq }}(\omega, q)$ in the complex frequency plane, i.e.,

$$
\begin{aligned}
& G_{\mathrm{eq}}(t, q)=\theta(t) H(q) e^{-i \mathfrak{w}_{0}(\epsilon, q) t}, \\
& \mathfrak{w}_{0}(\epsilon, q)=\epsilon^{z \nu} h\left(q \epsilon^{-\nu}\right),
\end{aligned}
$$

where $H(q)$ is some function that depends weakly on $q$. $h(x)$ is a universal scaling function that is analytic in $x^{2}$ for

\footnotetext{
${ }^{2}$ Independently, Sonner, del Campo, and Zurek [50] have found universal scaling behavior in the dynamics of strongly coupled superconductors with a holographic dual.
} 
small $x$. For $T>T_{c}, \mathfrak{w}_{0}(q, T)$ lies in the lower half $\omega$ plane, and its imaginary part at $q=0$ gives the inverse of the relaxation time. ${ }^{3}$ When continued to a supercooled state at $T<T_{c}, \mathfrak{w}_{0}$ moves to the upper half frequency plane for $q$ smaller than a certain $q_{\max }$, and for such $q$ 's, (2.5) grows exponentially with time. More explicitly, for positive $\epsilon$, we can expand $\operatorname{Im}_{0}$ in small $q$ as

$$
\operatorname{Imm}_{0}=-a \epsilon^{(z-2) \nu} q^{2}+b \epsilon^{z \nu}+\cdots,
$$

where $a$ and $b$ are positive constants. Hence, $\operatorname{Imm}_{0}>0$ until $q \sim q_{\max }$ with

$$
q_{\max } \sim \epsilon^{\nu} .
$$

Now, let us consider the limit of slow quenches $\epsilon^{\prime}(t) \rightarrow 0$. Assuming that the Green function depends weakly on temperature, then for a short interval $t-t^{\prime} \ll\left(1 /\left|\mathfrak{w}_{0}\right|\right)$ (2.5) should still apply, if $\mathfrak{w}_{0}$ changes with time sufficiently slowly, i.e.,

$$
\left|\partial_{t} \log \mathfrak{w}_{0}[T(t)]\right| \ll\left|\mathfrak{w}_{0}\right|
$$

Under this approximation, $G_{R}$ then satisfies a first-order differential equation

$$
\partial_{t} G_{R}\left(t, t^{\prime} ; \vec{k}\right)=-i \mathfrak{w}_{0}[T(t)] G_{R}\left(t, t^{\prime} ;\right)+\vec{k} \cdots, \quad t>t^{\prime},
$$

the integration of which leads to

$$
G_{R}\left(t, t^{\prime}, q\right)=\theta\left(t-t^{\prime}\right) H(q) e^{-i \int_{t^{\prime}}^{t} d t^{\prime \prime} \mathfrak{w}_{0}\left[\varepsilon\left(t^{\prime \prime}\right), q\right]} .
$$

From $\left|\partial_{t} \log \mathfrak{w}_{0}\right|<\left|\mathfrak{w}_{0}\right|$, and using (2.6) and (1.3), it is straightforward to show the earliest time when (2.10) can be applied is precisely $t>t_{\text {freeze }}$.

Substituting (2.10) into (2.4), we then secure

$$
C(t, q)=\int_{t_{\text {freeze }}}^{t} d t^{\prime} \zeta|H(q)|^{2} e^{2 \int_{t^{\prime}}^{t} d t^{\prime \prime} \operatorname{Im} \mathfrak{w}_{0}\left[\epsilon\left(t^{\prime \prime}\right), q\right]}+\cdots
$$

The ellipses in (2.11) denote the contributions in (2.4) coming from the integration domain $t^{\prime}<t_{\text {freeze }}$, which will be neglected in our discussion below as the first term in (2.11) grows exponentially with time and will soon dominate. ${ }^{4}$ We note that $\mathfrak{w}_{0}$ can also have a real part and therefore lead to oscillations of the order parameter

\footnotetext{
${ }^{3}$ In the language of the dual gravitational description discussed below, $\mathfrak{w}_{0}$ is the lowest quasinormal mode frequency of a dual black hole.

${ }^{4}$ In addition to the exponential suppression in time, when Fourier transformed to real space, the omitted terms in (2.11) also fall off parametrically faster with distance than the first term.
}

superimposed to the exponential growth induced by the imaginary part. These oscillations are an interesting phenomenon that deserves further discussion. Let us consider the behavior of the above integral for $t$ parametrically large compared to $t_{\text {freeze }}$ assuming for the moment that the linear analysis holds. For this purpose, it is convenient to introduce

$$
\bar{t} \equiv \frac{t}{t_{\text {freeze }}} .
$$

In the regime $\bar{t} \gg 1$, we find for $q \xi_{\text {freeze }} \ll 1$ (see Appendix A for details)

$$
C(t, q)=a_{1} \zeta t_{\text {freeze }} \exp \left[a_{2} \bar{t}^{1+\nu z}-\frac{1}{2} q^{2} \ell_{\mathrm{co}}^{2}(\bar{t})\right],
$$

where

$$
\ell_{\text {co }}(\bar{t})=a_{3} \xi_{\text {freeze }} \bar{t}^{[1+(z-2) \nu] / 2}
$$

and $a_{1}, a_{2}, a_{3}$ are $O\left(\tau_{Q}^{0}\right)$ constants. Fourier transforming $q$ to coordinate space, we find

$$
C(t, r) \sim|\psi|^{2}(t) e^{-r^{2} / 2 \ell_{\mathrm{co}}^{2}(t)}, \quad \text { with } \quad|\psi|^{2}(t) \sim \tilde{\varepsilon}(t) e^{a_{2} \tilde{t}^{1+z \nu}},
$$

where

$$
\tilde{\varepsilon}(t) \equiv \zeta t_{\text {freeze }} \ell_{\text {co }}^{-d}(t) \sim \varepsilon t_{\text {freeze }} \bar{t}^{-d[1+\nu(z-2)] / 2}
$$

with

$$
\varepsilon \equiv \zeta \xi_{\text {freeze }}^{-d} \sim \zeta \tau_{Q}^{-d \nu /(1+\nu z)} .
$$

Equations (2.13)-(2.17) are our main results of this section. We now proceed to discuss their physical meaning and physical implications.

\section{B. Equilibration time and density of defects}

With the usual inverse volume dependence, $\varepsilon$ defined in (2.17) may be interpreted as the effective parameter characterizing fluctuations for a spatial region of size $\xi_{\text {freeze }}$, while $\varepsilon t_{\text {freeze }}$ may be interpreted as the fluctuations accumulated over a time scale of order $O\left(t_{\text {freeze }}\right)$. In the limit of large $\tau_{Q}, \varepsilon$ goes to 0 , justifying the use of linear response. The linear response analysis should break down at some point, which can be estimated by comparing $|\psi|^{2}(t)$ in (2.15) with the equilibrium value of the condensate square. Recall that in equilibrium, the expectation value of an order parameter for reduced temperature $\epsilon \ll 1$ is characterized by a critical exponent $\beta$

$$
|\psi|_{\mathrm{eq}}^{2}(\epsilon) \sim \epsilon^{2 \beta} .
$$

Introducing a scale $t_{\mathrm{eq}}$ by requiring 


$$
|\psi|^{2}\left(t=t_{\mathrm{eq}}\right) \sim|\psi|_{\mathrm{eq}}^{2}\left[\epsilon\left(t_{\mathrm{eq}}\right)\right],
$$

we expect the linear analysis to break down for $t \sim t_{\mathrm{eq}}$. In particular, for $t \gtrsim t_{\text {eq }}$, we expect the condensate growth to transition from the exponential growth of (2.15) to the adiabatic growth governed by (2.18) with $\epsilon$ in (2.18) given by the time-dependent reduced temperature (1.2). Moreover, the system does not contain a well-defined number of topological defects until a well-defined condensate forms that necessarily lies outside the domain of linear response. Thus, $t_{\mathrm{eq}}$ is also the natural time scale to measure the density of topological defects.

To estimate $t_{\text {eq }}$, we have to solve Eq. (2.19) for $t_{\text {eq }} / t_{\text {freeze }}$. From (2.14)-(2.18), it is clear that this ratio, which determines the duration of the coarsening region, is controlled by the dimensionless parameter

$$
\begin{aligned}
R & \equiv \frac{\epsilon^{2 \beta}}{\tilde{\varepsilon}(t)} \simeq \frac{\tau_{Q}^{-2 \beta /(1+\nu z)}}{\varepsilon t_{\text {freeze }}} \sim \zeta^{-1} \tau_{Q}^{\Lambda /(1+\nu z)}, \\
\Lambda & \equiv(d-z) \nu-2 \beta
\end{aligned}
$$

When $R \lesssim O(1)$, we must have $t_{\text {eq }} \sim t_{\text {freeze }}$. In this case, there is no hierarchy of scales between $t_{\text {freeze }}$ and $t_{\text {eq }}$ and the condensate begins to grow adiabatically after $t_{\text {freeze }}$. In other words, in this case, our analysis reduces to the standard story of the KZM and the density of topological defects is given by (1.4). When $R \gg 1$, there is, however, a hierarchy between $t_{\text {eq }}$ and $t_{\text {freeze }}$, and (2.15) applies over a parametrically large interval of time during which the condensate grows with time exponentially, and the coarsening length $\ell_{\mathrm{co}}(t)$, which controls the typical size of a condensate droplet, grows with time as a power. In particular, in the limit $R \rightarrow \infty$, from (2.15), (2.19), and (2.14), we see

$$
\frac{t_{\mathrm{eq}}}{t_{\text {freeze }}} \sim(\log R)^{1 /(1+\nu z)}+\cdots \rightarrow \infty
$$

and

$$
\frac{\ell_{\mathrm{co}}\left(t_{\mathrm{eq}}\right)}{\xi_{\text {freeze }}} \sim(\log R)^{[1+(z-2) \nu /[2(1+z \nu)]}+\cdots \rightarrow \infty .
$$

Thus, for $R \gg 1$, a parametrically large amount of coarsening occurs before a well-defined condensate even forms. The density of topological defects of dimension $D$ is then [using (2.14)]

$$
\begin{aligned}
\rho\left(t_{\mathrm{eq}}\right) & \sim 1 / \ell_{\mathrm{co}}^{d-D}\left(t_{\mathrm{eq}}\right) \\
& \sim\left[\log \left(\zeta^{-1} \tau_{Q}^{\Lambda /(1+\nu z)}\right)\right]^{-\{(d-D)[1+(z-2) \nu]\} / 2(1+z \nu)} \rho_{\mathrm{KZ}} .
\end{aligned}
$$

As a result of early-time coarsening, the defect density $\rho$ is parametrically much smaller than the Kibble-Zurek prediction $\rho_{\mathrm{KZ}}$ and the standard $\mathrm{KZ}$ scaling is corrected by a logarithmic prefactor. Possible systems with $R \gg 1$ will be further discussed in the conclusion section.

We stress that the time dependence of (2.15) differs from the scaling behavior of standard coarsening physics [51], which applies only after the magnitude $|\psi|$ has achieved its equilibrium value. The possible importance of early-time coarsening physics in the KZM has recently also been discussed in Ref. [34], but it assumed the scaling behavior of standard coarsening physics and thus is not compatible with our result.

\section{Rapid quenches}

By decreasing $\tau_{Q}$ (while keeping $T_{i}, T_{f}$ fixed), eventually the scaling (2.23) for the defect density must break down. In standard KZ discussions, this breakdown should happen when $t_{f} \lesssim t_{\text {freeze }}$. Here, we point out that for systems with $t_{\text {eq }} \gg t_{\text {freeze }}$, the scaling (2.23) breaks down for $t_{f} \ll t_{\mathrm{eq}}$, and can happen even for $t_{f}$ parametrically much larger than $t_{\text {freeze }}$. This behavior is easy to understand; for $t_{\mathrm{eq}} \gg t_{f} \gg t_{\text {freeze }}$, since the system stays at $T_{f}$ after $t_{f}$, the growth of the condensate will largely be controlled by the unstable modes at $T_{f}$, and the defect density will be determined by $T_{f}$ rather than $\tau_{Q}$. We now generalize the above discussion of far-from-equilibrium coarsening to such a case, where Eq. (2.11) should be modified to

$C(t, q)=\int_{t_{\text {freeze }}}^{t} d t^{\prime} \zeta|H(q)|^{2} e^{2 \operatorname{Imm}_{0}\left(\epsilon_{f}, q\right)\left(t-t^{\prime}\right)}+\cdots$,

where as commented below (2.11), the ellipses denote contributions from earlier times that can be neglected in subsequent discussions. Note that $\mathfrak{w}_{0}\left(\epsilon_{f}, q\right)$ is now evaluated at $\epsilon_{f} \equiv\left(T_{c}-T_{f}\right) / T_{c}$, which results in a simple $e^{t}$ growth for any $\nu, z$ [compare with (2.13)]. Fourier transforming the above expression, then $C(t, r)$ can be written in a scaling form (see Appendix A for details)

$$
C(t, r) \sim \epsilon_{f}^{(d-z) \nu} \zeta f\left[\epsilon_{f}^{\nu z}\left(t-t_{\text {freeze }}\right), r \epsilon_{f}^{\nu}\right]
$$

for some scaling function $f$. For $\epsilon_{f}^{\nu z}\left(t-t_{\text {freeze }}\right) \gg 1$ and $r \epsilon_{f}^{\nu} \gg 1$ (assuming linear response still applies), $f$ can be obtained explicitly and one finds

$$
\begin{aligned}
C(t, r) & =|\psi|^{2}(t) e^{-r^{2} / 2 \ell_{\mathrm{co}}^{2}(t)}, \\
|\psi|^{2}(t) & \sim \epsilon_{f}^{(d-z) \nu} \zeta \exp \left[2 b\left(t-t_{\text {freeze }}\right) \epsilon_{f}^{\nu z}\right]
\end{aligned}
$$

with

$$
\ell_{\mathrm{co}}^{2}(t)=4 a\left(t-t_{\text {freeze }}\right) \epsilon_{f}^{\nu(z-2)} .
$$

Note that in comparing with (2.13) and (2.14), we see that both the logarithm of the condensate square and the coarsening length square grow linearly with time. 
Parallel to the earlier discussion, we postulate that the linear response analysis breaks down when the condensate squared obtained from (2.26) becomes comparable to $|\psi|_{\text {eq }}^{2}$. To estimate the time scale $t_{\text {eq }}$ when this breakdown happens, it is again convenient to introduce

$$
R_{f} \equiv \frac{\epsilon_{f}^{2 \beta}}{\zeta \epsilon_{f}^{(d-z) \nu}}=\zeta^{-1} \epsilon_{f}^{-\Lambda},
$$

and the criterion for linear response to apply for $\epsilon_{f}^{\nu z}\left(t-t_{\text {freeze }}\right) \gg 1$ is again $R_{f} \gg 1$. In particular, the equilibrium time $t_{\text {eq }}$ and the density of defects should be given by

$$
\begin{aligned}
t_{\text {eq }}-t_{\text {freeze }} & \sim \begin{cases}\epsilon_{f}^{-z \nu} & R_{f} \lesssim O(1) \\
\epsilon_{f}^{-\nu z} \log R_{f} & R_{f} \gg 1,\end{cases} \\
\rho & \sim \begin{cases}\epsilon_{f}^{(d-D) \nu} & R_{f} \lesssim O(1) \\
\epsilon_{f}^{(d-D) \nu} \log ^{-(d-D) / 2} R_{f} & R_{f} \gg 1 .\end{cases}
\end{aligned}
$$

Clearly, $\rho$ is independent of $\tau_{Q}$.

For very fast quenches, i.e., $t_{f} \ll t_{\text {freeze }}$, the whole quench from $T_{i}$ to $T_{f}$ will be nonadiabatic. In such a case, at the end of quench, the system will have correlation length $\xi_{i} \sim \xi_{\mathrm{eq}}\left(T_{i}\right)$ imprinted from the state before the starting of quench, and the scale $t_{\text {freeze }}$ is no longer relevant. But, the above discussion of far-from-equilibrium coarsening still applies with $t-t_{\text {freeze }}$ replaced by $t-t_{f}$.

\section{NUMERICAL RESULTS: OUT-OF-EQUILIBRIUM DYNAMIC OF A HOLOGRAPHIC SUPERFLUID}

In this section, we test the predictions of the previous section by constructing numerical solutions for the time evolution of a $(2+1)$-dimensional holographic superfluid after a quench across a second-order phase transition. In Appendix B, we give a detailed account of the gravity setup and technical details. Here, we discuss the main results.

\section{A. Predictions for holographic systems}

Many examples of field theories with gravity duals are now known in various spacetime dimensions, which essentially consist of elementary bosons and fermions interacting with non-Abelian gauge fields. The rank $N$ of the gauge group is mapped to the Newton constant $G_{N}$ of

\footnotetext{
${ }^{5}$ Strictly speaking, the above discussion applies to $\xi_{i}<$ $\xi_{\min }\left(T_{f}\right) \equiv q_{\max }^{-1}\left(T_{f}\right) \sim \epsilon_{f}^{-\nu}$. For $\xi_{i}>\xi_{\min }\left(T_{f}\right)$, unstable $q$ modes with $q^{-1}<\xi_{i}$ will be averaged out and only those modes with $q^{-1}>\xi_{i}$ can grow.
}

the bulk gravity such that $G_{N} \sim 1 / N^{2}$; the classical gravity approximation in the bulk thus corresponds to the large $N$ limit in the boundary theory. Finite temperature in the boundary system is described on the gravity side by a black hole. In the large $N$ limit, thermal and quantum fluctuations are suppressed by $1 / N^{2}$ and on the gravity side are encoded in quantum gravity effects induced from the black hole's Hawking radiation.

In this paper, we consider a holographic superfluid phase transition in two spatial dimension with relevant topological defects being pointlike vortices. In the large $N$ limit, the phase transition has mean-field critical exponents with $\nu=1 / 2, z=2, \beta=1 / 2$, and $\zeta$ in (2.1) of order $O\left(1 / N^{2}\right)$. For such a system, the predictions from the KZM for density of superfluid vortices read

$$
t_{\text {freeze }} \sim \tau_{Q}^{1 / 2}, \quad \xi_{\text {freeze }} \sim \tau_{Q}^{1 / 4}, \quad \rho_{\mathrm{KZ}} \sim \tau_{Q}^{-1 / 2} .
$$

Applying the discussion of the last section to such large $N$ theories, we can make the following predictions.

(1) For slow quenches, i.e., quenches with $t_{f} \gg t_{\text {eq }}$, with $d=2$ and mean-field exponents, Eq. (2.15) becomes $\left(\bar{t}=t / t_{\text {freeze }}\right)$

$$
\begin{aligned}
C(t, r) & \sim|\psi|^{2}(t) e^{-r^{2} / 2 \ell_{\mathrm{co}}^{2}(t)}, \\
|\psi|^{2}(t) & \sim \tilde{\varepsilon} t_{\text {freeze }} \bar{t} e^{a_{2} \bar{t}^{2}}, \\
\ell_{\mathrm{co}}(t) & \sim \xi_{\text {freeze }} \sqrt{\bar{t}} .
\end{aligned}
$$

Furthermore, from (2.20), we find $\Lambda=-1$ and thus

$$
R \sim \zeta^{-1} \tau_{Q}^{-1 / 2} \sim N^{2} \tau_{Q}^{-1 / 2} .
$$

In the large $N$ limit, we always have $R \gg 1$, and from (2.21) and (2.23),

$$
\frac{t_{\text {eq }}}{t_{\text {freeze }}} \sim \sqrt{\log \frac{N^{2}}{\sqrt{\tau_{Q}}}}
$$

and

$$
\rho \sim \frac{1}{\sqrt{\log \frac{N^{2}}{\sqrt{\tau_{Q}}}}} \rho_{\mathrm{KZ}} .
$$

(2) For rapid quenches, with mean-field exponents and $d=2$, Eq. (2.26) can be written as

$$
\begin{aligned}
C(t, r) & =|\psi|^{2}(t) e^{-r^{2} / 2 \ell_{\mathrm{co}}^{2}(t)}, \\
|\psi|^{2}(t) & \sim \zeta \exp \left[2 b\left(t-t_{\text {freeze }}\right) \epsilon_{f}\right]
\end{aligned}
$$

with

$$
\ell_{\mathrm{co}}^{2}(t)=4 a\left(t-t_{\text {freeze }}\right) .
$$



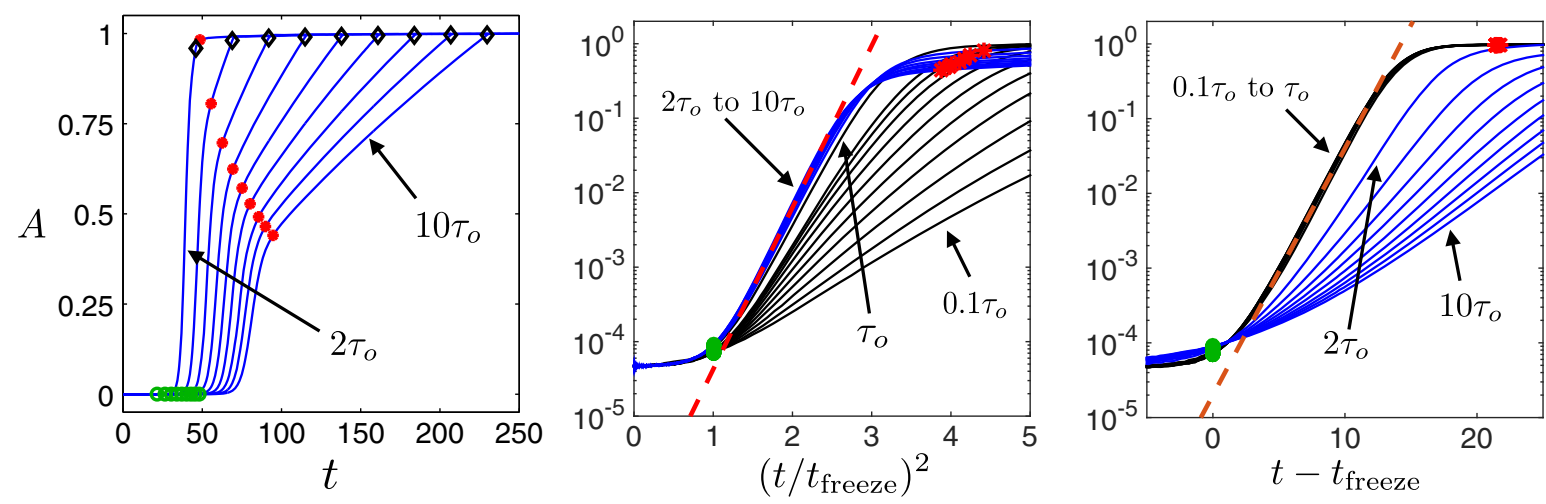

FIG. 1. Left: The normalized average condensate $A(t)$ defined in (3.9) for quench rates $\tau_{Q}=n \tau_{o}$ for $n=2, \ldots, 10$ (from left to right) with $\tau_{o}=161.37 / T_{c}$. The black diamonds denote the times at which for each $\tau_{Q}$ the thermal quench is over. All curves experience a period of rapid growth, which is followed by a period of approximate linear growth. The start of the rapid growth can be identified as $t_{\text {freeze }}$, which we operationally define as the time at which $A(t)=2 A(-\infty)$, and are denoted by the green circles. The crossover from exponential to linear growth corresponds to the equilibration time $t_{\mathrm{eq}}(2.19)$, which we operationally define as the time at which $A^{\prime \prime}\left(t_{\mathrm{eq}}\right)<0.1 \max \left\{A^{\prime \prime}(t)\right\}$, and are labeled by the red stars and circles. Middle: Scaling behavior of slow quenches; when $A$ is plotted vs $\bar{t}^{2}=\left(t / t_{\text {freeze }}\right)^{2}$, only curves corresponding to slow quenches collapse into a single one. The linear behavior in the logarithmic plot is consistent with (3.2). Right: Scaling behavior of rapid quenches; $A(t)$ is plotted vs $t-t_{\text {freeze }}$ on a logarithmic scale for quench rates $\tau_{Q}=n \tau_{o}$ for $n=0.1,0.2, \ldots, 10$. As predicted from (3.6), only $\tau_{Q}$ curves corresponding to fast quenches collapse into a single one, which exhibits linear exponential growth. All dimensional quantities are measured in units of $T_{c}(\mathrm{~B} 15)$.

Furthermore, $R_{f}=\zeta^{-1} \epsilon_{f}^{-1} \sim N^{2} \epsilon_{f}^{-1}$ is always much greater than 1 in the large $N$ limit, and we have from (2.29)

$$
t_{\text {eq }}-t_{\text {freeze }} \sim \epsilon_{f}^{-1} \log \frac{N^{2}}{\epsilon_{f}}, \quad \rho \sim \frac{\epsilon_{f}}{\log \frac{N^{2}}{\epsilon_{f}}} .
$$

Note that both quantities above are independent of $\tau_{Q}$.

\section{B. Numerical results}

We have performed numerical simulations of thermal quenches across a second-order phase transition of a $(2+1)$ dimensional holographic superfluid. We employ the linear quench (1.2), which in the gravity context translates into a black hole with a time-dependent temperature. Instead of directly computing fluctuations from Hawking radiation (see, e.g., Refs. [52,53]), we model fluctuations from quantum gravity effects as a random noise that enters as a nontrivial boundary condition in the gravity equation of motion. In such a formulation, $\zeta$ can be taken as an adjustable parameter, which we take to be numerically small so as to imitate the $O\left(1 / N^{2}\right)$ fluctuations. See Appendix B for details. In what follows, all dimensional quantities are expressed in units of the critical temperature $T_{c}$.

We begin our analysis by studying the normalized average order parameter

$$
A(t)=\frac{1}{M} \sum_{i=1}^{M} \frac{a_{i}(t)}{a_{i}(\infty)}, \quad a_{i}(t) \equiv \int d^{2} x\left|\psi_{i}(t, \boldsymbol{x})\right|^{2} .
$$

The sum over $i$ represents an ensemble average over $M$ configurations at fixed $\tau_{Q}$. The time evolution of $A(t)$ for various $\tau_{Q}$ are given in Fig. 1. The left and middle plots correspond to slow quenches where we see all curves experience a period of rapid growth after $t_{\text {freeze }}$ followed by a period of approximate linear growth. This regime of slow quenches is the one at which the $\mathrm{KZ}$ mechanism of defect formation applies. We note that the $\mathrm{KZ}$ mechanism assumes that defects are generated at $t_{\text {freeze }}$, so it does not provide a theory of the condensate growth. However, this problem has been previously addressed in the condensedmatter literature $[54,55]$. We operationally define $t_{\text {freeze }}$ as the time at which $A(t)=2 A(-\infty)$. The rapid growth can be
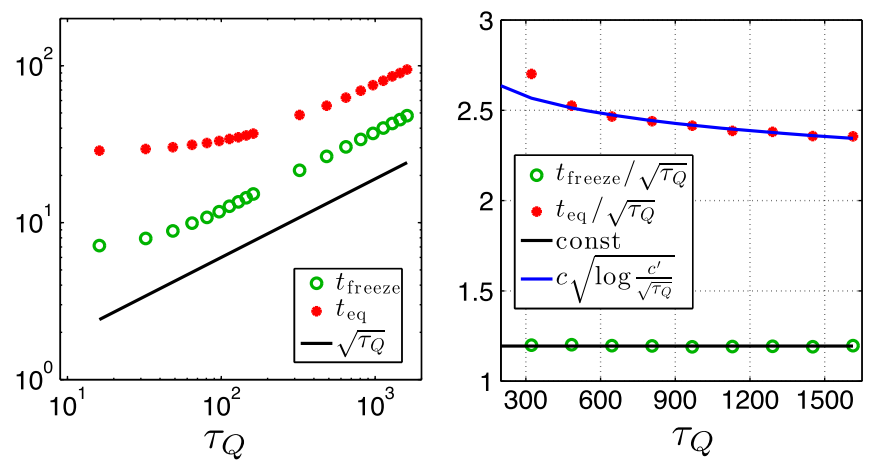

FIG. 2. Left: The freeze-out time $t_{\text {freeze }}$ and equilibration time $t_{\text {eq }}$ as a function of $\tau_{Q}$ expressed in units of $T_{c}$ (B15). For rapid quenches, $t_{\mathrm{eq}} \rightarrow$ const, as expected. For slow quenches, both $t_{\text {freeze }}$ and $t_{\mathrm{eq}}$ are consistent with the $\tau_{Q}^{1 / 2}$ scaling. Right: $t_{\mathrm{eq}}$ also exhibits logarithmic correction to the $\tau_{Q}^{1 / 2}$ scaling, consistent with the prediction of (3.4). 

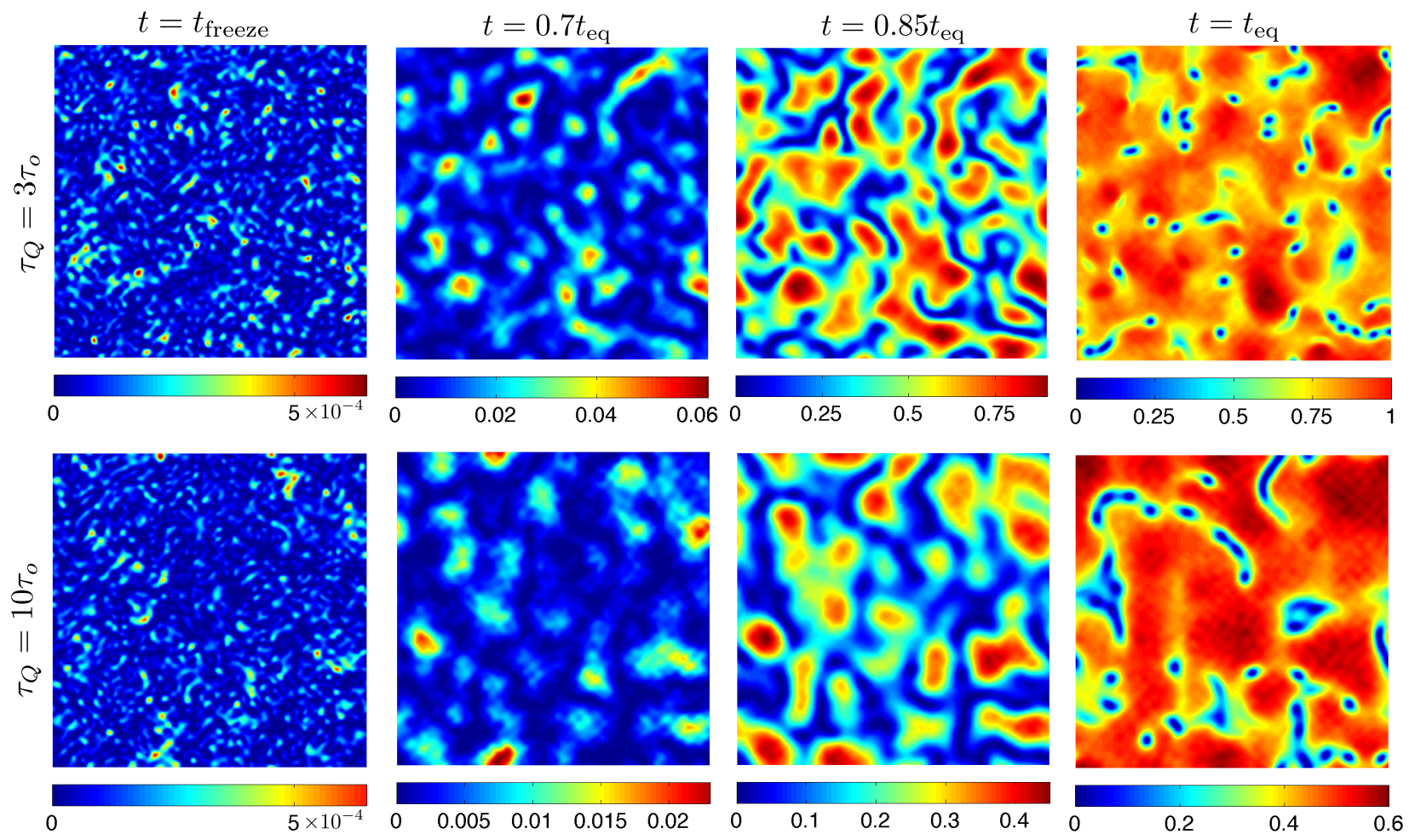

FIG. 3. The time evolution of $|\psi(t, \boldsymbol{x})|^{2} /|\psi(t=\infty, \boldsymbol{x})|^{2}$ for $\tau_{Q}=3 \tau_{o}$ (upper) and $\tau_{Q}=10 \tau_{o}$ (lower) at times $t=t_{\text {freeze }}, t=0.7 t_{\mathrm{eq}}$, $t=0.85 t_{\mathrm{eq}}$, and $t=t_{\mathrm{eq}}$. Similar results (not shown) are obtained for faster $\left(\tau_{Q} \leq \tau_{0}\right)$ quenches. The key message is that we can sensibly talk about defect density only after $t_{\mathrm{eq}}$. At $t=t_{\text {freeze }}$, the order parameter is very small and dominated by fluctuations. These fluctuations seed droplets of condensate, whose subsequent causal connection can be seen at time $t=0.7 t_{\mathrm{eq}}$. At such a time, the droplets are still separated by large regions where there is no condensate. Subsequently, the droplets expand and grow in amplitude and the system becomes smoother and smoother. By time $t_{\mathrm{eq}}$, the droplets have merged into a comparatively uniform condensate with isolated regions where $\psi=0$. The nonuniformities - the localized blue "dots"-are superfluid vortices with winding number \pm 1 . All dimensional quantities are expressed in units of $T_{c}$ (B15).
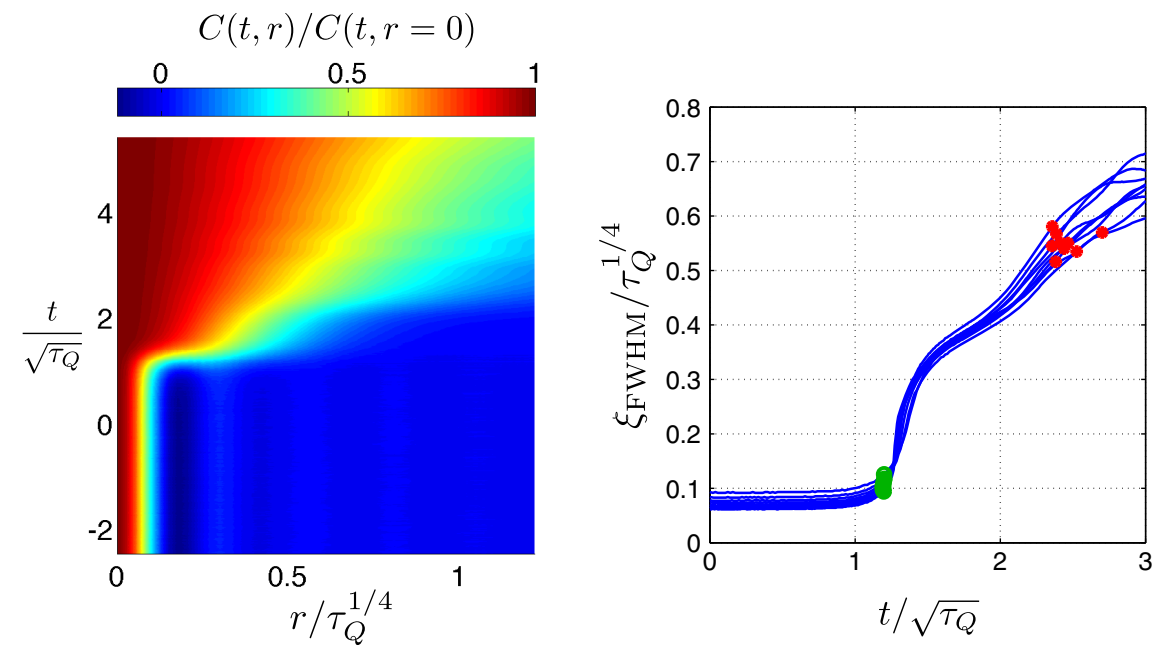

FIG. 4. Left: $C(t, r)$ for $\tau_{Q}=4 \tau_{o}$. Right: The time evolution of the full width at half maximum $\xi_{\mathrm{FWHM}}(t)$ of $C(t, r)$ for $\tau_{Q}=\tau_{o} n$ with $n=2,3,4, \ldots, 10$. The green circles correspond to $\xi_{\mathrm{FWHM}}\left(t_{\text {freeze }}\right)$ while the red stars correspond to $\xi_{\mathrm{FWHM}}\left(t_{\mathrm{eq}}\right)$. At $t \approx t_{\text {freeze }}, \xi_{\mathrm{FWHM}}$ starts a period of growth. Note that $\xi_{\mathrm{FWHM}}\left(t_{\text {freeze }}\right) \gg \xi_{\mathrm{FWHM}}\left(t_{\mathrm{eq}}\right)$. The collapse of all curves between $t_{\text {freeze }}$ and $t_{\mathrm{eq}}$ is consistent with the scaling behavior of (3.2). It is possible that the observed oscillations superimposed on the square-root growth of the $\xi_{\mathrm{FWHM}}(t)$ are related to the finite real part of $w_{0}(2.5)$. 
identified with the regime described by (2.15) and (3.2) as indicated by the middle plot. The linear growth can be identified as the regime of adiabatic condensate growth. To see this, note that for the mean field, $|\psi|_{\text {eq }}^{2} \sim \epsilon(t)=t / \tau_{Q}$, implying $A(t) \sim t / \tau_{Q}$ for adiabatic growth. This conclusion is supported by the slope of the linear growth, the observation that the termination of the linear condensate growth coincides with the end of the quench, and that when extrapolated to $t=0$, the linear curves have $A=0$. The crossover from exponential to linear growth corresponds to the equilibration time $t_{\text {eq }}$ (2.19), which we operationally defined as the time in which $A^{\prime \prime}\left(t_{\mathrm{eq}}\right)<0.1 \max \left\{A^{\prime \prime}(t)\right\}$.

A key feature of the middle plot of Fig. 1 is that curves of different $\tau_{Q}$ all lie top of one another when we plot them in terms of the scaling variable $\bar{t}^{2}=\left(t / t_{\text {freeze }}\right)^{2}$. In particular, the linear $\bar{t}^{2}$ growth in the logarithmic plot agrees very well with the prediction of (3.2). We note that in the context of condensed matter, the formation of a $1 D$ condensate was investigated numerically in Ref. [55]. Indeed, a similar collapse, for different $\tau_{Q}$ 's, was recently observed [8] in a $1 D$ system governed by the stochastic Gross-Pitaevskii equation. The right plot describes fast quenches discussed in Sec. IIC, with all the qualitative features of (3.6) confirmed numerically, namely, $e^{t}$ growth as compared with the $e^{t^{2}}$ growth of slow quenches, and all curves of different $\tau_{Q}$ lying on top of one another when plotted vs $t-t_{\text {freeze }}{ }^{6}$ For such a "rapid" quench, the growth of the condensate and the resulting defect density are dictated by $T_{f}$ and are independent of $\tau_{Q}$. This expectation is also borne out in Fig. 2, where we plot the freeze-out time $t_{\text {freeze }}$ and equilibration time $t_{\mathrm{eq}}$ as a function of $\tau_{Q}$. While for large $\tau_{Q}$, their behavior is consistent with $\sqrt{\tau_{Q}}$ scaling, for rapid quenches, $t_{\text {eq }}$ approaches a constant. The right panel of Fig. 2 also shows that our numerical results are consistent with the presence of a logarithmic hierarchy between the two time scales as predicted in (3.4).

In Fig. 3, we plot the time evolution of $|\psi(t, x)|^{2} /$ $|\psi(t=\infty, \boldsymbol{x})|^{2}$ for two values of $\tau_{Q}$ at various times up to $t=t_{\text {eq }}$. These plots help to visualize the key point that before $t_{\text {eq }}$ when a relative uniform $|\psi|^{2}$ has not formed, one cannot sensibly count defects. Moreover, it is evident that the defect density is higher for the faster quench.

To quantify the time evolution of coarsening and smoothing of the condensate, we numerically compute the correlation function $C(t, r)$ by computing the average in (2.3) over an ensemble of solutions at fixed $\tau_{Q}$. The results are in Fig. 4, where we also present the full width at half

\footnotetext{
${ }^{6}$ We note that our numerical results go at least up to times $t \sim 2 t_{\text {eq }}$. This is the shortest time scale necessary to distinguish between the growth corresponding to fast and slow quenches. For slow quenches, technical limitations related to long computation times prevent us from going beyond this time scale.
}

maximum $\xi_{\mathrm{FWHM}}(t)$ of $C(t, r)$. Before $t_{\text {freeze }}, \xi_{\mathrm{FWHM}}$ is dominated by fluctuations and is therefore constant. After $t \approx t_{\text {freeze }}, \xi_{\text {FWHM }}$ experiences a period of rapid growth, which is consistent with our prediction (3.2) including the scaling behavior. Note that $\xi_{\mathrm{FWHM}}\left(t_{\mathrm{eq}}\right)$ is significantly larger than $\xi_{\mathrm{FWHM}}\left(t_{\text {freeze }}\right)$, which highlights the importance of the fudge factor needed to account for the correct defect density. This observation is in line with our expectation from Eqs. (2.14) and (3.4).

Finally, in the left panel of Fig. 5, we show that for slow quenches, our numerical results reproduce the $\mathrm{KZ}$ scaling of the number of vortices $N_{\text {vortices. }}$. For $\tau_{Q}<200$, our numerics are consistent with $N_{\text {vortices }}=$ const. A constant number of vortices is the expected behavior from our discussion of the breakdown of the KZ scaling in the preceding section: The density of defects should asymptote to a constant in the limit of sudden quenches. For such rapid quenches, the right plot confirms the scaling of the defect density with $\epsilon_{f}$ as predicted in (2.29) and (3.8). For both situations, our statistics are not enough to resolve the logarithms predicted in (3.5) and (3.8). Also included in the left panel of Fig. 5 is a plot of $\left[L B / \xi_{\mathrm{FWHM}}\left(t_{\mathrm{eq}}\right)\right]^{2}$, where $B \approx 1.92$. The fantastic agreement between $\left[L B / \xi_{\mathrm{FWHM}}\left(t_{\mathrm{eq}}\right)\right]^{2}$ and $N_{\text {vortices }}$ for all $\tau_{Q}$ bolsters the notion that the vortex density is a measure of the correlation length. Moreover, the observation that $B=O(1)$ and $\xi_{\text {FWHM }}\left(t_{\text {eq }}\right) \gg \xi_{\text {FWHM }}\left(t_{\text {freeze }}\right)$ is consistent with our argument that coarsening during the early stages of the
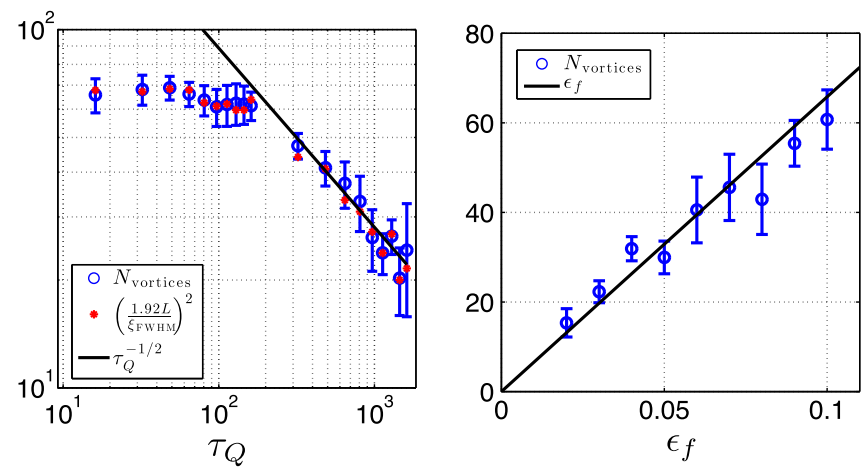

FIG. 5. Left: The number of vortices $N_{\text {vortices }}$ and $\xi_{\text {FWHM }}^{-2}$ at time $t=t_{\text {eq }}$ as a function of $\tau_{Q}$ expressed in units of $T_{c}$ (B15). Each data point is computed by averaging the number of vortices over an ensemble of solutions at fixed $\tau_{Q}$. The error bars are computed from the variance of $N_{\text {vortices. }}$. The numerical results are consistent with $\mathrm{KZ}$ scaling $\tau_{Q}^{-1 / 2}$ for $\tau_{Q}>200$. For $\tau_{Q}<200$, our numerics are consistent with $N_{\text {vortices }}=$ const, which is consistent with our expectation that the density of defects should asymptote to a constant in the limit of sudden quenches. Also included is a plot of $\left[L B / \xi_{\mathrm{FWHM}}\left(t_{\mathrm{eq}}\right)\right]^{2}$, where $L$ is our box size and $B \approx 1.92$. Our statistics are not sufficient to resolve the logarithmic prefactor in (3.5). Right: $N_{\text {vortices }}$ vs $\epsilon_{f}=\epsilon\left(T_{f}\right)$, in units of $T_{c}$ (B15) for sufficiently small $\tau_{Q}$. The results are consistent with (3.8) with $N_{\text {vortices }} \sim \epsilon_{f}$. Our statistics are again not sufficient to resolve the logarithmic prefactor in (3.8). 
evolution can dramatically increase the correlation length and decrease the expected density of defects from the KZ prediction (1.4).

\section{CONCLUSION AND DISCUSSION}

To summarize, we elucidated a novel period of nonadiabatic evolution after a system passes through a secondorder phase transition, where a parametrically large amount of coarsening occurs before a well-defined condensate forms. The physical origin of the coarsening can be traced to the fact that when the system passes through the phase transition, IR modes of the order parameter become unstable and exponentially grow. We showed that such a far-from-equilibrium coarsening regime could have important consequences for defect formation. We also numerically simulated thermal quenches in a $(2+1)$-dimensional holographic superfluid, which provided strong support for our analytic results.

For slow quenches, a key quantity is $R(2.20)$, which we copy here for convenience:

$$
R \sim \zeta^{-1} \tau_{Q}^{\Lambda /(1+\nu z)}, \quad \Lambda \equiv(d-z) \nu-2 \beta .
$$

For $R \gg 1$, there is a large hierarchy between $t_{\text {freeze }}$ and $t_{\mathrm{eq}}$, and the density of defects can be significantly lower than that predicted by KZ. Systems with $R \gg 1$ can be separated into two cases.

(I) The exponent $\Lambda$ is positive, i.e.,

$$
(d-z) \nu>2 \beta
$$

for which $R \rightarrow \infty$ as $\tau_{Q} \rightarrow \infty$. For a mean field with $z=2, \nu=\frac{1}{2}, \beta=\frac{1}{2}$, Eq. (4.2) implies $d>4$, i.e., above the upper critical dimension of mean-field theory. Using the hyperscaling relation $2 \beta=$ $(d-2+\eta) \nu$, for a general critical point, $\Lambda$ can be simplified as

$$
\Lambda=(2-\eta-z) \nu
$$

and (4.2) becomes

$$
z<2-\eta
$$

An example that satisfies this condition is superfluid ${ }^{4} \mathrm{He}$, which has

$$
z=\frac{3}{2}, \quad \eta \approx 0.037 .
$$

Other examples include a three-dimensional isotropic antiferromagnet and the three-dimensional $X Y$ model.

(II) $\Lambda$ is negative, but $\zeta \ll 1$, so that for large but finite $\tau_{Q}$, we still have $R \gg 1$, i.e.,

$$
\zeta \ll \tau_{Q}^{\Lambda /(1+\nu z)}
$$

One class of examples is holographic theories, such as that discussed in Sec. III, for which $\zeta \sim 1 / N^{2}$ with $N \rightarrow \infty$. As another class of examples, let us consider model $A$ for dynamic critical phenomena [56]. Recall that the van Hove theory of critical slowing-down predicted exactly $z=2-\eta$. Renormalization-group analysis gives $z$ slightly greater than this value [57], which means that generically for model $A, \Lambda$ is only slightly negative, and thus (4.6) essentially translates into $\zeta \ll 1$. As an explicit example, conventional superconductors have a very small $\zeta$, and thus we expect them to have a large hierarchy between $t_{\text {freeze }}$ and $t_{\mathrm{eq}}$.

For fast quenches, the analogous quantity is $R_{f}$ defined in (2.28). Comparing (2.28) with (2.20), we see the conditions for $R_{f} \gg 1$ are essentially identical to those for $R \gg 1$, and the above discussion also applies.

\section{ACKNOWLEDGMENTS}

We thank Laurence Yaffe for useful discussions. The work of P. M. C. is supported by the Fundamental Laws Initiative of the Center for the Fundamental Laws of Nature at Harvard University. The work of H. L. is partially supported by the U.S. Department of Energy (DOE) under Cooperative Research Agreement No. DEFG0205ER41360. A. M. G.-G. was supported by Engineering and Physical Sciences Research Council, Grant No. EP/I004637/1; Fundação para a Ciência e a Tecnologia, Grant No. PTDC/FIS/111348/2009; and a Marie Curie International Reintegration Grant No. PIRG07-GA-2010-268172.

\section{APPENDIX A: DETAILS ON VARIOUS INTEGRALS}

In this section, we give some details in the derivation of (2.13) and (2.26).

\section{Slow quenches}

Consider the integral (2.11) for small $q \lesssim q_{\max }$

$$
\begin{aligned}
C(t, q)= & \int_{t_{\text {freeze }}}^{t} d t^{\prime} \zeta|H(q)|^{2} e^{2 \int_{t^{\prime}}^{t} d t^{\prime \prime} \operatorname{Imw}_{0}\left[\epsilon\left(t^{\prime \prime}\right), q\right]} \\
\approx & \zeta|H(0)|^{2} t_{\text {freeze }} \int_{1}^{\bar{t}} d \overline{t^{\prime}} \\
& \times \exp \left[2 t_{\text {freeze }} \int_{\bar{t}^{\prime}}^{\bar{t}} d \overline{t^{\prime \prime}} \operatorname{Imm}_{0}\left[\epsilon\left(t^{\prime \prime}\right), q\right]\right],
\end{aligned}
$$

where we have introduced $\bar{t}=t / t_{\text {freeze }}$. Now, note from (2.6) that

$$
t_{\text {freeze }} \operatorname{Imm}_{0}[\epsilon(t), q]=-\frac{a \tau_{s}}{\xi_{s}^{2}} \bar{t}^{(z-2) \nu} q^{2} \xi_{\text {freeze }}^{2}+b \tau_{s} \bar{t}^{z \nu}+\cdots,
$$


where we have used (1.2) and (1.3). We thus find that

$$
\begin{aligned}
C(t, q) & \approx \zeta C|H(0)|^{2} t_{\text {freeze }} \exp \left[a_{2} \bar{t}^{1+\nu z}-\frac{1}{2} q^{2} \ell_{\mathrm{co}}^{2}(\bar{t})\right], \\
\ell_{\mathrm{co}}^{2}(\bar{t}) & \equiv a_{3}^{2} \xi_{\text {freeze }}^{2} \bar{t}^{1+(z-2) \nu}
\end{aligned}
$$

where

$$
\begin{aligned}
a_{2} & =\frac{2 b \tau_{s}}{1+z \nu}, \quad a_{3}^{2}=\frac{4 a \tau_{s}}{[1+\nu(z-2)] \xi_{s}^{2}}, \\
C & =\int_{1}^{\bar{t}} d x \exp \left(-a_{1} x^{1+\nu z}+\frac{1}{2} a_{3}^{2} q^{2} \xi_{\text {freeze }}^{2} x^{1+\nu(z-2)}\right) .
\end{aligned}
$$

Note that since $x>1, \nu>0$, and we are interested in the regime $q \xi_{\text {freeze }} \lesssim O(1)$, the first term in the exponential of $C$ always dominates over the second term. For large $\bar{t}$, due to exponential suppression, the integral for $C$ is dominated by the lower end, and we thus conclude that $C \sim O(1)$. For $\bar{t} \sim O(1)$, the $\bar{t}$ dependence is more complicated, but this is not the regime we are interested in. Suppressing various $O(1)$ prefactors, we thus find (2.13) and (2.14).

Note that in (2.11), we have assumed the condensate starts growing at $t_{\text {freeze }}$, but it is clear from our derivation that (2.13) and (2.14) are not sensitive to the specific time when the condensate starts growing. For example, the conclusion remains the same if the lower end of the integral of (A1) is changed to 0 .

\section{Rapid quenches}

For rapid quenches discussed in Sec. II C, we have

$$
\begin{aligned}
C(t, q) & =\int_{t_{\text {freeze }}}^{t} d t^{\prime} \zeta|H(q)|^{2} e^{2 \operatorname{Imm} \mathfrak{w}_{0}\left(\epsilon_{f}, q\right)\left(t-t^{\prime}\right)} \\
& \approx \frac{\zeta|H(0)|^{2}}{\operatorname{Im} \mathfrak{w}_{0}\left(\epsilon_{f}, q\right)}\left[e^{2 \operatorname{Imm}_{0}\left(\epsilon_{f}, q\right)\left(t-t_{\text {freeze }}\right)}-1\right] .
\end{aligned}
$$

Given the scaling form of $\operatorname{Imm}_{0}(T, q)=\epsilon^{\nu z} \operatorname{Im} h\left(q \epsilon^{-\nu}\right)$, the above equation can be written as

$$
\begin{aligned}
C(t, q) & \approx \frac{\epsilon_{f}^{-\nu z} \zeta|H(0)|^{2}}{2 \operatorname{Im} h(\tilde{q})}\left[e^{2 \operatorname{Im} h(\tilde{q}) \tilde{t}}-1\right], \quad \tilde{q} \equiv q \epsilon_{f}^{-\nu}, \\
\tilde{t} & \equiv\left(t-t_{\text {freeze }}\right) \epsilon_{f}^{\nu z}
\end{aligned}
$$

whose Fourier transform can also be written in a scaling form

$$
C(t, r)=\epsilon_{f}^{(d-z) \nu} \zeta f(\tilde{t}, \tilde{r}), \quad \tilde{r} \equiv r \epsilon_{f}^{\nu}
$$

for some scaling function $f$. For large $\tilde{r} \gg 1$, we can use the small $\tilde{q}$ expansion $h(\tilde{q})=b-a \tilde{q}^{2}$, and for $\tilde{t} \gg 1$, we find that

$$
C(t, r) \sim \epsilon_{f}^{(d-z) \nu} \zeta \exp \left[2 b\left(t-t_{\text {freeze }}\right) \epsilon_{f}^{\nu z}-\frac{r^{2}}{2 \ell_{\mathrm{co}}^{2}(t)}\right]
$$

with

$$
\ell_{\mathrm{co}}^{2}(t)=4 a\left(t-t_{\text {freeze }}\right) \epsilon_{f}^{\nu(z-2)} .
$$

\section{APPENDIX B: NONEQUILIBRIUM HOLOGRAPHIC SUPERFLUIDITY: GRAVITY SETUP}

The field content of the $(2+1)$-dimensional holographic superfluid we study consists of the metric $G_{M N}$, a $U(1)$ gauge field $A^{M}$, and a charged scalar $\Phi$ with charge $e$. These fields live in asymptotically $\mathrm{AdS}_{4}$ spacetime. Following Ref. [58], we take the action to be

$$
\begin{aligned}
S_{\text {grav }}= & \frac{1}{16 \pi G_{\text {Newton }}} \int d^{4} x \sqrt{-G} \\
\times & {\left[R+\Lambda+\frac{1}{e^{2}}\left(-\frac{1}{4} F_{M N} F^{M N}\right.\right.} \\
& \left.\left.\quad-|D \Phi|^{2}-m^{2}|\Phi|^{2}\right)\right],
\end{aligned}
$$

where $R$ is the Ricci scalar, $F_{M N}$ is the $U(1)$ field strength, $D$ is the gauge-covariant derivative, and $G=-\operatorname{det} G_{M N}$. The mass $m$ of the scalar field and the cosmological constant $\Lambda$ are given by

$$
m^{2}=-2, \quad \Lambda=-3 .
$$

The $U(1)$ gauge redundancy in the bulk encodes a $U(1)$ global symmetry in the boundary theory where the boundary order parameter transforms with a phase $\psi \rightarrow \psi e^{i \alpha}$. Indeed, the bulk scalar field $\Phi$ encodes $\psi$.

Following Ref. [38], we employ infalling EddingtonFinkelstein coordinates where the metric takes the form

$$
d s^{2}=r^{2} g_{\mu \nu}(t, \boldsymbol{x}, r) d x^{\mu} d x^{\nu}+2 d r d t .
$$

Here, greek indices run over boundary spacetime coordinates and $r$ is the anti-de Sitter (AdS) radial coordinate with $r=\infty$ the AdS boundary. With our choice of coordinates, lines of constant $t$ represent infalling null radial geodesics affinely parametrized by $r$. In addition, we choose to work in the gauge $A_{r}=0$.

For simplicity, we choose to work in the probe limit $e \rightarrow \infty$ where gravitational dynamics decouple from the dynamics of the gauge and scalar fields. The equations of motion following from (B1) are then simply

$$
0=R_{M N}-\frac{1}{2} G_{M N}(R+2 \Lambda),
$$




$$
\begin{aligned}
& 0=\nabla_{M} F^{N M}-J^{M}, \\
& 0=\left(-D^{2}+m^{2}\right) \Phi .
\end{aligned}
$$

Since the boundary of AdS is timelike, the equations of motion (B4) require boundary conditions to be imposed there. As the boundary geometry of AdS corresponds to the geometry the dual quantum theory lives in, we demand that the boundary geometry be that of flat $(2+1)$-dimensional Minkowski space. This is accomplished by setting $\lim _{r \rightarrow \infty} g_{\mu \nu}=\eta_{\mu \nu}$. The near-boundary behavior of the gauge and scalar fields can easily be worked by from Eqs. (B4b) and $(\mathrm{B} 4 \mathrm{c})$ and read

$$
\begin{aligned}
& A_{\alpha}(t, \boldsymbol{x}, r)=A_{\alpha}^{(0)}(t, \boldsymbol{x})+\frac{A_{\alpha}^{(1)}(t, \boldsymbol{x})}{r}+O\left(1 / r^{2}\right), \\
& \Phi(t, \boldsymbol{x}, r)=\frac{\Phi_{(1)}(t, \boldsymbol{x})}{r}+\frac{\Phi_{(2)}(t, \boldsymbol{x})}{r^{2}}+O\left(1 / r^{3}\right) .
\end{aligned}
$$

On the gauge field, we impose the boundary condition

$$
A_{\alpha}^{(0)}(t, \boldsymbol{x})=\delta_{\alpha 0} \mu,
$$

where $\mu$ is a constant. In the dual quantum field theory, $\mu$ is interpreted as a chemical potential for the conserved $U(1)$ charge. As a final boundary condition, we set

$$
\Phi_{(1)}(t, \boldsymbol{x})=\varphi(t, \boldsymbol{x})
$$

with $\varphi$ random variable satisfying statistics (2.1). The stochastic driving of the scalar field mimics the effect of quantum and thermal fluctuations induced by the black brane's Hawking radiation. In the dual quantum theory, the boundary condition (B8) amounts to deforming the Hamiltonian

$$
H \rightarrow H+\int d^{2} x\left\{\varphi^{*} \psi+\varphi \psi^{*}\right\} .
$$

Note that $\varphi$ has mass dimension one and $\psi$ has mass dimension two. In terms of the asymptotic behavior of the scalar field (B6), the boundary order parameter reads

$$
\psi(t, \boldsymbol{x})=\Phi_{(2)}(t, \boldsymbol{x})-\left(\partial_{t}-i \mu\right) \varphi(t, \boldsymbol{x}) .
$$

Let us first discuss static equilibrium solutions to the set of equations of motion (B4). Translationally invariant equilibrium solutions to Einstein's equations consist of black branes

$$
d s^{2}=r^{2}\left[-f d t^{2}+d \boldsymbol{x}^{2}\right]+2 d r d t,
$$

where

$$
f=1-\left(\frac{r_{h}}{r}\right)^{3} .
$$

The Hawking temperature $T$ of the black brane is related to the horizon radius $r_{h}$ by

$$
r_{h}=\frac{4 \pi T}{3}
$$

and corresponds to the temperature of the dual quantum theory.

Static equilibrium solutions to the scalar-gauge-field system (B4c) and (B4b) were first explored in Ref. [58]. One static solution to (B4c) and (B4b) (with $\varepsilon=0$ and hence no stochastic driving) is simply

$$
\begin{gathered}
A_{\alpha}=\mu\left(1-\frac{r_{h}}{r}\right) \delta_{\alpha 0}, \\
\Phi=0 .
\end{gathered}
$$

However, for sufficiently low temperatures, this solution is unstable and not thermodynamically preferred. For $T<T_{c}$, where

$$
T_{c} \approx 0.0587 \mu,
$$

the thermodynamically preferred solution has $\Phi \neq 0$. Hence, the bulk $U(1)$ gauge redundancy is spontaneously broken at low temperatures and the black brane develops a charged scalar atmosphere. Likewise, via (B10), the boundary order parameter is nonzero and the global $U(1)$ symmetry on the boundary is spontaneously broken. The gravitational and boundary systems have a second-order phase transition at $T=T_{c}$ with mean-field critical exponents.

To study the Kibble-Zurek mechanism gravitationally, we drive the system stochastically with the boundary condition (B8) and choose to dynamically cool the black brane geometry through $T_{c}$. When the geometry cools through $T_{c}$, the aforementioned instability will result in the scalar field $\Phi$ growing and the black brane developing a scalar atmosphere. Likewise, as this cooling happens, the boundary quantum field theory condensate (B10) will grow in amplitude.

Instead of solving Einstein's equations (B4a) for a black brane with dynamic temperature, we choose to fix the geometry to be the equilibrium geometry (B11) but with a time-dependent temperature $T(t)$ equal to the boundary quench protocol temperature (1.2), which we control. The metric will therefore no longer satisfy Einstein's equations. Why is it reasonable to employ a geometry that does not satisfy Einstein's equations? To answer this question, we note that to cool the system through $T_{c}$, we can couple it to an external thermal bath at controllable temperature $T_{\text {ext }}(t)$. This coupling can be done by, for example, putting our 
system in a box of size $L$ and putting the surface of the box in contact with the thermal reservoir. As we are ultimately interested in slow quenches where $T_{\text {ext }}^{\prime}(t)$ is parametrically small, we expect thermal equilibration and $T(t) \approx T_{\text {ext }}(t)$. In this limit, Einstein's equations can be solved with the gradient expansion of fluid and gravity [59]. At leading order in gradients, the solution is simply (B11), but with the time-dependent temperature $T(t)$.

Our numerical methods used to solve the scalar-gaugefield system (B4c) and (B4b) are outlined in Ref. [38]. We use pseudospectral methods and discretize the AdS radial coordinate using 20 Chebyshev polynomials. In the spatial directions, we work in a periodic spatial box and discretize using a basis of 201 plane waves. We chose box size $L T_{c}=$ 30.8 and measure all other dimensionful quantities in units of $T_{c}$. We choose noise amplitude $\zeta T_{c}=1.5 \times 10^{-3}$. As our quench protocol (1.2) starts off at temperatures $T>T_{c}$, in the infinite past, we choose initial conditions (B14).

[1] T. W. B. Kibble, Topology of Cosmic Domains and Strings, J. Phys. A 9, 1387 (1976).

[2] T. W. B. Kibble, Some Implications of a Cosmological Phase Transition, Phys. Rep. 67, 183 (1980).

[3] W.H. Zurek, Cosmological Experiments in Superfluid Helium? Nature (London) 317, 505 (1985).

[4] P. Laguna and W. H. Zurek, Density of Kinks after a Quench: When Symmetry Breaks, How Big Are the Pieces? Phys. Rev. Lett. 78, 2519 (1997).

[5] J. Dziarmaga, J. Meisner, and W. H. Zurek, Winding Up of the Wave-Function Phase by an Insulator-to-Superfluid Transition in a Ring of Coupled Bose-Einstein Condensates, Phys. Rev. Lett. 101, 115701 (2008).

[6] S.-W. Su, S.-C. Gou, A. Bradley, O. Fialko, and J. Brand, Kibble-Zurek Scaling and Its Breakdown for Spontaneous Generation of Josephson Vortices in Bose-Einstein Condensates, Phys. Rev. Lett. 110, 215302 (2013).

[7] H. Saito, Y. Kawaguchi, and M. Ueda, Kibble-Zurek Mechanism in a Quenched Ferromagnetic Bose-Einstein Condensate, Phys. Rev. A 76, 043613 (2007).

[8] B. Damski and W. H. Zurek, Soliton Creation during a BoseEinstein Condensation, Phys. Rev. Lett. 104, 160404 (2010).

[9] A. Das, J. Sabbatini, and W. H. Zurek, Winding Up Superfluid in a Torus via Bose Einstein Condensation, Sci. Rep. 2, 352 (2012).

[10] M. Kolodrubetz, B. K. Clark, and D. A. Huse, Nonequilibrium Dynamic Critical Scaling of the Quantum Ising Chain, Phys. Rev. Lett. 109, 015701 (2012).

[11] L. Wang, C. Zhou, T. Tu, H.-W. Jiang, G.-P. Guo, and G.-C. Guo, Quantum Simulation of the Kibble-Zurek Mechanism Using a Semiconductor Electron Charge Qubit, Phys. Rev. A 89, 022337 (2014).

[12] X.-Y. Xu, Y.-J. Han, K. Sun, J.-S. Xu, J.-S. Tang, C.-F. Li, and G.-C. Guo, Quantum Simulation of Landau-Zener Model Dynamics Supporting the Kibble-Zurek Mechanism, Phys. Rev. Lett. 112, 035701 (2014).

[13] A. del Campo, G. De Chiara, G. Morigi, M. B. Plenio, and A. Retzker, Structural Defects in Ion Chains by Quenching the External Potential: The Inhomogeneous Kibble-Zurek Mechanism, Phys. Rev. Lett. 105, 075701 (2010).

[14] L. Mathey and A. Polkovnikov, Light Cone Dynamics and Reverse Kibble-Zurek Mechanism in Two-Dimensional Superfluids Following a Quantum Quench, Phys. Rev. A 81, 033605 (2010).

[15] W. H. Zurek, U. Dorner, and P. Zoller, Dynamics of a Quantum Phase Transition, Phys. Rev. Lett. 95, 105701 (2005).

[16] J. Dziarmaga, Dynamics of a Quantum Phase Transition: Exact Solution of the Quantum Ising Model, Phys. Rev. Lett. 95, 245701 (2005).

[17] B. Damski, The Simplest Quantum Model Supporting the Kibble-Zurek Mechanism of Topological Defect Production: Landau-Zener Transitions from a New Perspective, Phys. Rev. Lett. 95, 035701 (2005).

[18] A. Chandran, A. Erez, S. S. Gubser, and S. L. Sondhi, Kibble-Zurek Problem: Universality and the Scaling Limit, Phys. Rev. B 86, 064304 (2012).

[19] K. Pyka, J. Keller, H. L. Partner, R. Nigmatullin, T. Burgermeister, D. M. Meier, K. Kuhlmann, A. Retzker, M. B. Plenio, W. H. Zurek et al., Topological Defect Formation and Spontaneous Symmetry Breaking in Ion Coulomb Crystals, Nat. Commun. 4, 2291 (2013).

[20] S. Ulm, J. Roßnagel, G. Jacob, C. Degünther, S. T. Dawkins, U. G. Poschinger, R. Nigmatullin, A. Retzker, M. B. Plenio, F. Schmidt-Kaler et al., Observation of the Kibble-Zurek Scaling Law for Defect Formation in Ion Crystals, Nat. Commun. 4, 2290 (2013).

[21] C. N. Weiler, T. W. Neely, D. R. Scherer, A. S. Bradley, M. J. Davis, and B. P. Anderson, Spontaneous Vortices in the Formation of Bose-Einstein Condensates, Nature (London) 455, 948 (2008).

[22] D. R. Scherer, C. N. Weiler, T. W. Neely, and B. P. Anderson, Vortex Formation by Merging of Multiple Trapped BoseEinstein Condensates, Phys. Rev. Lett. 98, 110402 (2007).

[23] G. Lamporesi, S. Donadello, S. Serafini, F. Dalfovo, and G. Ferrari, Spontaneous Creation of Kibble-Zurek Solitons in a Bose-Einstein Condensate, Nat. Phys. 9, 656 (2013).

[24] I. Chuang, B. Yurke, R. Durrer, and N. Turok, Cosmology in the Laboratory-Defect Dynamics in Liquid Crystals, Science 251, 1336 (1991).

[25] A. Maniv, E. Polturak, and G. Koren, Observation of Magnetic Flux Generated Spontaneously during a Rapid Quench of Superconducting Films, Phys. Rev. Lett. 91, 197001 (2003).

[26] K. G. Lagoudakis, F. Manni, B. Pietka, M. Wouters, T. C. H. Liew, V. Savona, A. V. Kavokin, R. André, and B. DeveaudPlédran, Probing the Dynamics of Spontaneous Quantum Vortices in Polariton Superfluids, Phys. Rev. Lett. 106, 115301 (2011).

[27] R. Carmi, E. Polturak, and G. Koren, Observation of Spontaneous Flux Generation in a Multi-JosephsonJunction Loop, Phys. Rev. Lett. 84, 4966 (2000).

[28] V. M. H. Ruutu, V. B. Eltsov, A. J. Gill, T. W. B. Kibble, M. Krusius, Y. G. Makhlin, B. Placais, G. E. Volovik, and W. $\mathrm{Xu}$, Vortex Formation in Neutron-Irradiated Superfluid ${ }^{3} \mathrm{He}$ as an Analogue of Cosmological Defect Formation, Nature (London) 382, 334 (1996).

[29] V. M. Ruutu, V. B. Eltsov, M. Krusius, Y. G. Makhlin, B. Plaçais, and G.E. Volovik, Defect Formation in 
Quench-Cooled Superfluid Phase Transition, Phys. Rev. Lett. 80, 1465 (1998).

[30] C. Bäuerle, Y. M. Bunkov, S. N. Fisher, H. Godfrin, and G. R. Pickett, Laboratory Simulation of Cosmic String Formation in the Early Universe Using Superfluid ${ }^{3} \mathrm{He}$, Nature (London) 382, 332 (1996).

[31] W. H. Zurek, Cosmological Experiments in Condensed Matter Systems, Phys. Rep. 276, 177 (1996).

[32] J. Dziarmaga, Dynamics of a Quantum Phase Transition and Relaxation to a Steady State, Adv. Phys. 59, 1063 (2010).

[33] A. del Campo and W. H. Zurek, Universality of Phase Transition Dynamics: Topological Defects from Symmetry Breaking, Int. J. Mod. Phys. A 29, 1430018 (2014).

[34] G. Biroli, L. F. Cugliandolo, and A. Sicilia, Kibble-Zurek Mechanism and Infinitely Slow Annealing through Critical Points, Phys. Rev. E 81, 050101 (2010).

[35] J. Maldacena, The Large-n Limit of Superconformal Field Theories and Supergravity, Int. J. Theor. Phys. 38, 1113 (1999).

[36] E. Witten, Anti-de Sitter Space and Holography, Adv. Theor. Math. Phys. 2, 253 (1998).

[37] S. S. Gubser, I. R. Klebanov, and A. M. Polyakov, Gauge Theory Correlators from Noncritical String Theory, Phys. Lett. B 428, 105 (1998).

[38] P. M. Chesler and L. G. Yaffe, Numerical Solution of Gravitational Dynamics in Asymptotically Anti-de Sitter Spacetimes, arXiv:1309.1439.

[39] J. Casalderrey-Solana, M. P. Heller, D. Mateos, and W. van der Schee, Longitudinal Coherence in a Holographic Model of p-Pb Collisions, Phys. Rev. Lett. 112, 221602 (2014).

[40] A. Adams, P. M. Chesler, and H. Liu, Holographic Vortex Liquids and Superfluid Turbulence, Science 341, 368 (2013).

[41] H. Bantilan, F. Pretorius, and S. S. Gubser, Simulation of Asymptotically $\mathrm{AdS}_{5}$ Spacetimes with a Generalized Harmonic Evolution Scheme, Phys. Rev. D 85, 084038 (2012).

[42] P. M. Chesler and L. G. Yaffe, Holography and Colliding Gravitational Shock Waves in Asymptotically $\mathrm{AdS}_{5}$ Spacetime, Phys. Rev. Lett. 106, 021601 (2011).

[43] R. A. Janik, AdS/CFT for the Early Stages of Heavy Ion Collisions, Nucl. Phys. A931, 176 (2014).

[44] P. M. Chesler and L. G. Yaffe, Horizon Formation and Far-from-Equilibrium Isotropization in Supersymmetric Yang-Mills Plasma, Phys. Rev. Lett. 102, 211601 (2009).
[45] K. Murata, S. Kinoshita, and N. Tanahashi, Non-equilibrium Condensation Process in a Holographic Superconductor, J. High Energy Phys. 07 (2010) 50.

[46] M. J. Bhaseen, J. P. Gauntlett, B. D. Simons, J. Sonner, and T. Wiseman, Holographic Superfluids and the Dynamics of Symmetry Breaking, Phys. Rev. Lett. 110, 015301 (2013).

[47] A. M. García-García, H. B. Zeng, and H.-Q. Zhang, A Thermal Quench Induces Spatial Inhomogeneities in a Holographic Superconductor, J. High Energy Phys. 07 (2014) 96.

[48] S. R. Das, Holographic Quantum Quench, J. Phys. Conf. Ser. 343, 012027 (2012).

[49] P. Basu, D. Das, S. R. Das, and K. Sengupta, Quantum Quench and Double Trace Couplings, J. High Energy Phys. 12 (2013) 070.

[50] J. Sonner, A. del Campo, and W. H. Zurek, Universal Farfrom-Equilibrium Dynamics of a Holographic Superconductor, arXiv:1406.2329.

[51] A. J. Bray, Theory of Phase-Ordering Kinetics, Adv. Phys. 43, 357 (1994).

[52] S. Caron-Huot, P. M. Chesler, and D. Teaney, Fluctuation, Dissipation, and Thermalization in Nonequilibrium $\mathrm{AdS}_{5}$ Black Hole Geometries, Phys. Rev. D 84, 026012 (2011).

[53] P. M. Chesler and D. Teaney, Dilaton Emission and Absorption from Far-from-Equilibrium Non-Abelian Plasma, arXiv:1211.0343.

[54] H. T. C. Stoof, Coherent versus Incoherent Dynamics during Bose-Einstein Condensation in Atomic Gases, J. Low Temp. Phys. 114, 11 (1999).

[55] H. T. C. Stoof and M. J. Bijlsma, Dynamics of Fluctuating Bose-Einstein Condensates, J. Low Temp. Phys. 124, 431 (2001).

[56] P. C. Hohenberg and B. I. Halperin, Theory of Dynamic Critical Phenomena, Rev. Mod. Phys. 49, 435 (1977).

[57] R. Folk and G. Moser, Critical Dynamics: A FieldTheoretical Approach, J. Phys. A 39, R207 (2006).

[58] S. A. Hartnoll, C. P. Herzog, and G. T. Horowitz, Building a Holographic Superconductor, Phys. Rev. Lett. 101, 031601 (2008).

[59] S. Bhattacharyya, S. Minwalla, V. E Hubeny, and M. Rangamani, Nonlinear Fluid Dynamics from Gravity, J. High Energy Phys. 02 (2008) 045. 